\title{
Cosmological status of Lagrangian theory of density perturbations
}

\author{
V. Strokov ${ }^{1,2 *}$ \\ 1 Astro Space Center of the P. N. Lebedev Physical Institute of RAS \\ 117997 Moscow, ul. Profsoyuznaya, 84/32 \\ 2 Moscow Institute of Physics and Technology, Department of General and Applied Physics \\ 141701 Dolgoprudny, Institutskiy per., 9
}

\begin{abstract}
We show that hydrodynamical and field approaches in theory of cosmological scalar perturbations are equivalent for a single medium. We also give relations between notations introduced by V. Lukash, J. Bardeen, J. Bardeen et al. and G. Chibisov and V. Mukhanov.
\end{abstract}

PACS: 98.80.-k, 98.62.Ai

*e-mail: strokov@asc.rssi.ru 


\section{Introduction}

In the linear theory of primordial cosmological perturbations the metric tensor $g_{\mu \nu}$ and energymomentum tensor of matter $T_{\mu \nu}$ are splitted into a background part and a perturbation part: $g_{\mu \nu}=g_{\mu \nu}^{(0)}+h_{\mu \nu}, T_{\mu \nu}=T_{\mu \nu}^{(0)}+\delta T_{\mu \nu}$. Evidently, this splitting into background and perturbation is ambiguous. Making small coordinate transformations we obtain a different background and a different perturbation. And in a given reference frame we can get "perturbations" which are actually not physical and are just a result of the chosen reference frame. In order to avoid such phantom perturbations, formalism of gauge-invariant variables is used. Equations which describe the evolution of a gauge-invariant variable come from the perturbed part of Einstein equations and properties of matter. Clearly, Einstein equations solely are insufficient to solve the dynamical problem as they just relate perturbations of the metric tensor with those of the energy-momentum tensor without specifying any physics. In order to obtain a dynamical equation we need a physical relation. For example, it can be a relation between matter density and pressure perturbations which corresponds to the hydrodynamical approach. Also we can start from a general matter Lagrangian of $\varphi$-field (which serves as a 4-velocity potential of matter), and this corresponds to the field approach. The former is usually associated with equation of state of matter, while the latter is with models of inflation. In either case it allows one to get dynamics and a Lagrangian of perturbations which appear to be independent on the initial assumptions.

Further we set out the main results of the Lagrangian theory of scalar perturbations [1] without derivation. We show the equivalence of hydrodynamical and field approaches for a single medium. Then we show how notations of Lukash [1, Bardeen [2], Bardeen et al. [3] and Chibisov and Mukhanov 4] are related.

\section{Gauge-invariant formalism in a nutshell}

Below we work with the background Friedmann-Robertson-Walker geometry:

$$
\begin{gathered}
d s^{2}=d t^{2}-a^{2} d x_{i} d x^{i}=a^{2}\left(d \eta^{2}-d x_{i} d x^{i}\right), \\
T_{\mu \nu}=(\varepsilon+p) u_{\mu} u_{\nu}-p g_{\mu \nu} .
\end{gathered}
$$

The background metric tensor is $g_{\mu \nu}=\operatorname{diag}\left(1,-a^{2},-a^{2},-a^{2}\right)$, matter density $\varepsilon$ and pressure $p$ are functions of time, and the background 4-velocity is $u^{\mu}=(1,0,0,0)$, the speed of light $c=1$, and $\eta$ is conformal time, $d \eta=d t / a$. Hereafter we omit the superscript (0) for background quantities.

The basic equations for the scale factor $a(t)$ are the Friedmann equations:

$$
\begin{gathered}
H^{2}=\frac{8 \pi G}{3} \varepsilon, \\
\gamma \equiv-\frac{\dot{H}}{H^{2}}=\frac{3}{2}\left(1+\frac{p}{\varepsilon}\right),
\end{gathered}
$$


where $H=\dot{a} / a=a^{\prime} / a^{2}$ is the Hubble parameter, and $G$ is the gravitational constant. The dot and the prime stand for the derivative with respect to physical time $t$ and conformal time $\eta$, respectively.

Generally, scalar type metric perturbations are constructed using four potentials [5], $A, B, C$ and $D$ :

$$
h_{\mu \nu}=\left(\begin{array}{cc}
2 D & C_{, i} \\
C_{, i} & 2 a^{2}\left(A \delta_{i j}+B_{, i j}\right)
\end{array}\right) .
$$

The potential $A$ is actually a perturbation of the scale factor: $A=-\delta a / a$.

Perturbation of the energy-momentum tensor is presented via other four potentials, $v, \delta \varepsilon, \delta p$ and $E$ :

$$
\begin{gathered}
\delta T_{0}^{0}=\delta \varepsilon, \\
\delta T_{i}^{0}=(\varepsilon+p) v_{, i}, \\
-\delta T_{j}^{i}=\delta p \delta_{i j}+(\varepsilon+p) \sigma_{i j}, \\
\sigma_{i j}=\frac{1}{2 a^{2} H^{2}}\left(E_{, i j}-\triangle E \delta_{i j}\right), \quad \sigma_{i, j}^{j}=0,
\end{gathered}
$$

where $E$ presents anisotropic stresses, and $v$ is the 3 -velocity potential:

$$
u_{\mu}=\left(1+D, v_{, i}\right) .
$$

Thus, we have four gravitational potentials $A, B, C, D$ and four matter potentials $v, \delta \varepsilon, \delta p, E$. All of them but $E$ are not gauge-invariant. By small coordinate transformations $x^{\mu} \rightarrow x^{\mu}+\xi^{\mu}$ the potentials get changed. Two of these eight potentials are arbitrary, they correspond to a gauge choice (an arbitrary vector in scalar representation $\xi_{\mu}=F u_{\mu}+H_{, \mu}$ ). It is possible to construct some gauge-invariant combinations of the potentials. All such combinations constitute an infinite set.

The potentials $A, B, C, D, v, \delta \varepsilon, \delta p, E$ are not independent. They are linked through the first-order expansion of the Einstein equations

$$
\delta G_{\nu}^{\mu}=8 \pi G \delta T_{\nu}^{\mu} .
$$

The natural gauge-invariant combination is that of the gravitational potential $A$ and the velocity potential $v$ which is called the $q$-scalar [1:

$$
q=A+H v .
$$

The inverse transformations of the $q$-field to the original potentials are as follows:

$$
v=\frac{q-A}{H}, \quad \delta p_{c} \equiv \delta p-\dot{p} v=\frac{\varepsilon+p}{H} \dot{q},
$$




$$
\begin{gathered}
a^{2} \dot{B}-C=\frac{A-\Phi}{H}, \quad D=\gamma q-\frac{d}{d t}\left(\frac{A}{H}\right), \\
\delta \varepsilon_{c} \equiv \delta \varepsilon-\dot{\varepsilon} v=\frac{\triangle \Phi}{4 \pi G a^{2}}, \quad \Phi=\frac{H}{a} \int a \gamma(q+E) d t,
\end{gathered}
$$

where $\delta p_{c}$ and $\delta \varepsilon_{c}$ are gauge-invariant variables of pressure and energy density perturbation, respectively. The first equation in (11) is, in fact, the relativistic Poisson equation. From the inverse transformations it can explicitly be seen that $\Phi$ and $E$ are gauge-invariant potentials.

The previous analysis is common and does not depend on any matter physics. However, in order to introduce dynamics we need some additional relation between matter quantities (e.g. between $\delta p_{c}$ and $\delta \varepsilon_{c}$ ), that is, we need to specify some physics. We have two possibilities: either we can use the hydrodynamical approach to relate $\delta p_{c}$ and $\delta \varepsilon_{c}$ or the field approach, i.e. to admit some form of the matter Lagrangian for 4-velocity potential of the medium. Further it is shown that the both approaches are equivalent in this problem. Further on we consider a single medium. Also, we suppose absence of anisotropic stresses, therefore,

$$
E=0, \quad \Phi=\frac{H}{a} \int a \gamma q d t .
$$

Hydrodynamical approach. In the hydrodynamical approach we assume

$$
\delta p_{c}=\beta^{2}(t) \delta \varepsilon_{c},
$$

where $\beta(t)$ is a function of time. Hence from (9) and (2),

$$
\delta \varepsilon_{c}=\alpha^{2} H \dot{q}, \quad \alpha^{2}=\frac{\gamma}{4 \pi G \beta^{2}} .
$$

Relation (13) means that there is only one medium and we describe its perturbations. As soon as (13) is valid equations (11) and (14) immediately give:

$$
\gamma \beta^{-2} a^{3} \dot{q}=\int a \gamma \triangle q d t .
$$

After differentiation the last equation gives equation describing the evolution of $q$-scalar:

$$
\ddot{q}+\left(3 H+2 \frac{\dot{\alpha}}{\alpha}\right) \dot{q}-\left(\frac{\beta}{a}\right)^{2} \triangle q=0 .
$$

The equation (16) corresponds to the action [1]

$$
\begin{aligned}
S[q] & =\frac{1}{2} \int \alpha^{2}\left(\dot{q}^{2}-\left(\frac{\beta}{a}\right)^{2} q_{, i} q^{, i}\right) a^{3} d t d^{3} x= \\
& =\frac{1}{2} \int(\alpha a)^{2}\left({q^{\prime}}^{2}-\beta^{2} q_{, i} q^{, i}\right) d \eta d^{3} x .
\end{aligned}
$$


Since the backward path from equation to a Lagrangian defines the Lagrangian to a factor before it, we can see that (17) has the right coefficient if we look at it in some asymptotic limit, e.g. in the limit of small scales (the sound wave frequency $\omega \gg H$ and sound velocity $c_{s} \simeq \beta$ ). In this approximation $q \simeq H v, \dot{q} \simeq H \dot{v}$ and $\delta \varepsilon_{c} \simeq \delta \varepsilon$. Using the relations:

$$
\frac{\delta \varepsilon}{\varepsilon+p}=\frac{\dot{v}}{c_{s}^{2}}, \quad \frac{\nabla v}{a}=-\mathbf{v},
$$

where $\mathbf{v}$ is hydrodynamical velocity in a sound wave, we have the following chain of equalities:

$$
\begin{gathered}
L[q]=\frac{1}{2}(a \alpha)^{2}\left({q^{\prime}}^{2}-\beta^{2} q_{, i} q^{, i}\right)=\frac{1}{2}(\alpha a H)^{2}\left({v^{\prime}}^{2}-c_{s}^{2}(\nabla v)^{2}\right)= \\
=\frac{a^{4}}{2}\left(c_{s}^{2} \frac{\delta \varepsilon^{2}}{\varepsilon+p}-(\varepsilon+p) \mathbf{v}^{2}\right) .
\end{gathered}
$$

The corresponding comoving volume energy density is

$$
\mathcal{E}=\frac{1}{2}\left(c_{s}^{2} \frac{\delta \varepsilon^{2}}{\varepsilon+p}+(\varepsilon+p) \mathbf{v}^{2}\right) .
$$

The last expression is exactly the energy density in a sound wave [6, 1 .

Field approach. The Universe filled with a scalar field $\varphi$. The relation to the 4 -velocity (6) is

$$
u_{\mu}=\frac{\varphi, \mu}{w},
$$

where $w^{2}=\varphi_{, \mu} \varphi_{, \nu} g^{\mu \nu}$. The Lagrangian density of the scalar field can be taken in a quite arbitrary form [1, 9]:

$$
\mathcal{L}=\mathcal{L}(\varphi, w) .
$$

From the matter Lagrangian (22) we obtain the energy-momentum tensor:

$$
\varepsilon=n w-\mathcal{L}, \quad p=\mathcal{L}, \quad n \equiv \frac{\partial \mathcal{L}}{\partial w}, \quad v=\frac{\delta \varphi}{\dot{\varphi}}
$$

and hence, the following relation between $\delta \varepsilon_{c}$ and $\delta p_{c}$ :

$$
\frac{\delta \varepsilon_{c}}{\delta p_{c}}=\frac{n_{, w} w \delta w-n_{, w} \dot{w} \delta \varphi}{n \delta w-n \dot{w} w^{-1} \delta \varphi}=\frac{n_{, w} w}{n}=\frac{w}{n} \frac{\partial^{2} \mathcal{L}}{\partial w^{2}} \equiv c_{s}^{-2}(\varphi, w) .
$$

For the linear perturbations the function $c_{s}^{-2}(\varphi, w)$ is taken in the zero order and turns into a function of time $c_{s}^{-2}=c_{s}^{-2}(t)$. This proves that the background functions are identical, $c_{s}=\beta$, and, thus, both ways, (13) and (22), of deriving equation (16) are identical as well.

In the field approach one can obtain the Lagrangian describing perturbations by expanding straightforwardly the action for gravitating scalar field to the second order in perturbation. The action is standard:

$$
S\left[\varphi, g_{\mu \nu}\right]=\int\left(\mathcal{L}-\frac{1}{16 \pi G} R\right)(-g)^{1 / 2} d^{4} x,
$$


where $R$ is scalar curvature. Perturbing the variables to the linear order, $g_{\mu \nu} \rightarrow g_{\mu \nu}+h_{\mu \nu}, \varphi \rightarrow \varphi+w v$, and decomposing (25) up to the second order terms we obtain the action for perturbations (total divergency terms are omitted):

$$
\begin{aligned}
& \delta^{(2)} S\left[v, h_{\mu \nu}\right]=-\frac{1}{64 \pi G} \int\left(\bar{h}_{\sigma \beta ; \alpha} \bar{h}^{\sigma \beta ; \alpha}-2 \bar{h}_{; \sigma}^{\alpha \beta} \bar{h}_{\alpha ; \beta}^{\sigma}-\frac{1}{2} \square \bar{h}\right)(-g)^{1 / 2} d^{4} x- \\
& -\frac{1}{4} \int\left(\frac{1}{16 \pi G} R-\mathcal{L}\right)\left(\bar{h}_{\nu}^{\mu} \bar{h}_{\mu}^{\nu}-\frac{1}{2} \bar{h}^{2}\right)(-g)^{1 / 2} d^{4} x+ \\
& +\frac{1}{2} \int n w\left[\nu v \bar{h}-2 u_{\mu} v_{\nu} \bar{h}^{\mu \nu}+\chi^{2}\left(c_{s}^{-2}-1\right)+m^{2} v^{2}+2 \Gamma v \chi\right](-g)^{1 / 2} d^{4} x, \\
& n \nu=-\frac{\partial \mathcal{L}}{\partial \varphi}, \quad v_{\mu}=\frac{(w v)_{, \mu}}{w}, \quad \chi=\frac{\delta w}{w}, \quad m^{2}=-\frac{w}{n} \frac{\partial^{2} \mathcal{L}}{\partial \varphi^{2}}, \quad \Gamma=\frac{w}{n} \frac{\partial^{2} \mathcal{L}}{\partial w \partial \varphi} .
\end{aligned}
$$

Here $\bar{h}_{\mu \nu}=h_{\mu \nu}-\frac{1}{2} g_{\mu \nu} h$, is the so-called tensor with inverse trace: $\bar{h}=\bar{h}_{\sigma}^{\sigma}=-h=-h_{\sigma}^{\sigma}$. The operations of raising and lowering indices are performed using the background metric $g_{\mu \nu}$.

The variable $q$ is so remarkable, because after linking all the potentials through equations (9) (10) (11) and substituting the $q$-scalar (86) to the expansion (26) we get a very simple perturbation action (17) (totally divergent terms are excluded), where $q$ enters as a test massless-like field.

\section{Relation between $q, \zeta$ and $\Psi$}

Variables $\zeta$ and $\Phi_{H}$ were introduced in Bardeen [2] and Bardeen et al. 3]. They are expressed through $q$ and $\Phi$ as follows:

$$
\zeta=\frac{2}{3} \frac{H^{-1} \dot{\Phi}+\Phi}{1+w_{B}}+\Phi=\frac{1}{a \gamma} \frac{d}{d t}\left(\frac{a \Phi}{H}\right)=q, \quad \Phi_{H}=\Phi,
$$

where (cf. (3) )

$$
w_{B}=\frac{p}{\varepsilon}, \quad \gamma=\frac{3}{2}\left(1+w_{B}\right) .
$$

Obviously $\zeta$ coincides with $q$ introduced in a general form by equation (8).

In the work [7] the dynamical equation (equation (5.22) in [7]) was derived in variables $\Psi$ and $v^{(g i)}$. In those notations the variable $q$ [1] looks as follows:

$$
q=\Psi+H v^{(g i)},
$$

where

$$
\Psi=A-\frac{\left(B^{\prime}-C\right) a^{\prime}}{a}, \quad v^{(g i)}=v+a\left(B^{\prime}-C\right) .
$$

The identity of (28) and (8) is obvious if we substitute (29) to (28). 


\section{Conclusion}

The theory of scalar cosmological perturbations can be constructed in a quite general form (eqs. (11)(11)). In order to get the Lagrangian for perturbations and the key dynamical equation we needed the physical assumptions to link $\delta p_{c}$ and $\delta \varepsilon_{c}$. Moreover, the assumption (13) is equivalent to the assumption of a matter field $\varphi$ with a quite common Lagrangian $\mathcal{L}(\varphi, w)$, where $\varphi$ is a 4 -velocity potential of the medium (cf. (하) and (21)). In linear theory of a single gravitating medium the two approaches coincide.

The variable $\zeta$ introduced by Bardeen et al. 3 is equal to the variable $q$ introduced by Lukash [1, and equation (5.22) found by Mukhanov et al. [7] is equivalent to (16).

The author is grateful to V. N. Lukash, E. V. Mikheeva and P. B. Ivanov for fruitful discussions. The work was supported by UNK FIAN and Grant 04-02-17444 of Russian Foundation for Basic Research. 


\section{References}

[1] V. Lukash, Zh. Eksp. Teor. Fiz. 79, 1601 (1980).

[2] J. Bardeen, Phys. Rev. D 22, 1882 (1980).

[3] J. Bardeen, P. Steinhardt, and M. Turner, Phys. Rev. D 28, 679 (1983).

[4] G. Chibisov and V. Mukhanov, Mon. Not. R. Astron. Soc. 200, 535 (1982).

[5] L. Landau and E. Lifshitz, Field Theory, Nauka, Moscow, 1967.

[6] L. Landau and E. Lifshitz, Hydrodynamics, Nauka, Moscow, 1986.

[7] V. Mukhanov, H. Feldman, and R. Brandenberger, Physics Reports, 5 and 6, 203 (1992).

[8] H. Kodama and M. Sasaki, Inter. J. Mod. Phys. A 1, 265 (1986); A 2, 491 (1987).

[9] V. Lukash. The very early universe. Lectures. arXiv.org e-Print archive, astro-ph/9910009.

[10] E.M. Lifshitz, Zh. Eksp. Teor. Fiz. 16587 (1946). 